\title{
Treatment of selected canine dermatological conditions in Portugal - a research survey
}

\author{
Ana Oliveira ${ }^{1}$, Joana S.P. Devesa ${ }^{1}$, Peter B. Hill ${ }^{2}$, \\ Vanessa Silva ${ }^{3,4}$, Patrícia Poeta ${ }^{3,4}$ \\ ${ }^{1}$ Faculty of Veterinary Medicine, \\ University Lusófona de Humanidades e Tecnologias, 1749-024 Lisboa, Portugal \\ ${ }^{2}$ School of Animal and Veterinary Sciences, University of Adelaide, Adelaide, South Australia, 5371 Australia \\ ${ }^{3}$ Department of Veterinary Sciences, Escola de Ciências Agrárias e Veterinárias, \\ University of Trás-os-Montes and Alto Douro (UTAD), 5001-801 Vila Real, Portugal \\ ${ }^{4}$ Associated Laboratory for Green Chemistry (LAQV-REQUIMTE), \\ University NOVA of Lisboa, 1099-085 Lisboa, Caparica, Portugal \\ ana.dermatology@gmail.com
}

Received: May 24, $2018 \quad$ Accepted: October 16, 2018

\begin{abstract}
Introduction: Staphylococcus pseudintermedius and Malassezia pachydermatis often cause skin diseases in dogs. Material and Methods: An online survey was e-mailed to veterinary practices nationwide covering demographics, diagnosis methods, and oral and topical treatment options. Of the 740 surveys sent, 100 complete replies were obtained. Results: The majority of clinicians were unaware of the existence of the International Society for Companion Animal Infectious Diseases guidelines or did not follow them (53\%). Oral antibiotics were used universally for superficial bacterial folliculitis treatment, particularly amoxicillin-clavulanic acid (100\%), cephalexin (94\%), enrofloxacin (67\%), or marbofloxacin (60\%). For fold dermatitis (FD) and otitis externa $(\mathrm{OE})$, oral antibiotics were also given as treatment in $88 \%$ and $82 \%$ of cases, respectively. Oral antifungals were often prescribed for generalised Malassezia dermatitis (85\%), FD (70\%), and OE (59\%). S. pseudintermedius and M. pachydermatis were frequently treated topically, particularly with antibacterials or antifungals only, or a combination of antibacterials, antifungals, and glucocorticoids. Alternative options such as honey-based products were not frequently used. Conclusion: Our survey suggests that oral antibiotics are overused by Portuguese clinicians despite the spread of antibiotic resistant $S$. pseudintermedius. Oral antibiotics and antifungals are commonly prescribed for skin conditions manageable with topical treatments.
\end{abstract}

Keywords: dogs, Staphylococcus pseudintermedius, Malassezia pachydermatis, antibacterials, antifungals, survey.

\section{Introduction}

Canine pyoderma, particularly superficial bacterial folliculitis (SBF), and otitis externa (OE), are common reasons for veterinary consultation. Staphylococcus pseudintermedius, although normally isolated from healthy skin, mucosa, and ear canals, can also act as an opportunist pathogen. It is invariably associated with $\mathrm{SBF}$, as well as commonly found in cases of $\mathrm{OE}(1,21$, $16,18)$.

Malassezia pachydermatis is a normal inhabitant of canine skin and ears, although, when an adequate environment is created, it can also act as an opportunistic pathogen. This yeast commonly causes Malassezia dermatitis (MD) in dogs and is frequently associated with canine OE $(21,24,27)$. Skin infections can be treated with antibiotics and topical antiseptics like chlorhexidine $(5,18)$. Bacterial culture and antibiotic susceptibility testing increases the likelihood of prescribing the correct antibiotic $(5,8)$. An increase in the proliferation of antibiotic resistance has led to the demand for alternative treatments, for example with natural products, preferably to which microorganisms cannot acquire resistance. Widespread occurrences of methicillin-resistant S. pseudintermedius (MRSP) are well documented, and resistance to azoles in 
M. pachydermatis isolates has also been reported (8, $10,14,20,22,28)$.

This study had three objectives: first, to evaluate the current practice in Portugal regarding diagnosis and treatment of SBF, fold dermatitis (FD), and bacterial OE caused by $S$. pseudintermedius and compare it with the International Society for Companion Animal Infectious Diseases (ISCAID) recommendations. Secondly, to investigate the diagnostic methodology and treatment for dermatitis, FD, and $\mathrm{OE}$ caused by $M$. pachydermatis, and finally, to determine if alternative topical products, namely medical honey, are being used in the management of these conditions.

\section{Material and Methods}

Survey. An 18-question survey was developed in Google Forms to interrogate practitioner approach to the diagnosis and management of skin and ear infections associated with $S$. pseudintermedius or $M$. pachydermatis in dogs. Two questions were designed to assess diagnostic approaches, 13 questions addressed treatment choices, and three questions covered participant demographics. The questions were designed to avoid bias by being multiple-choice and permitting only one option to be selected (with the exception of one question).

An e-mail was sent nationwide in October 2017 through Mailchimp software (the Rocket Science Group, Atlanta, USA) with the link to the survey. It was directed to 740 veterinary hospitals and clinics located in Portugal and was intentionally limited to only one survey per practice.

\section{Results}

Total replies. From the total of 740 e-mails, we obtained 103 replies (a 14\% response rate). Three surveys were incomplete and thus excluded. A total of 100 replies were considered valid.

Demographics. Fifty-two respondents were located in the centre of the country, 31 were from the south and 15 from the northern regions. One response came from the Azores and one from the Madeira archipelago. Thirteen percent of respondents had been in practice for less than 5 years, $26 \%$ between 5 and 10 years, $42 \%$ between 10 and 20 years, and $19 \%$ had more than 20 years of clinical experience.

Use of the ISCAID guidelines. About a third $(32 \%)$ of the respondents applied the ISCAID guidelines for the diagnosis and treatment of SBF in practice. Most participants were not aware of the guidelines $(53 \%)$ or did not apply them in practice (15\%).

Diagnosis. Of the four conditions surveyed, the prevalence was highest for OE. On a monthly basis, all clinicians diagnosed at least one case of OE (100\%). Malassezia dermatitis was the next disease most commonly seen disease $(81 \%)$, followed by FD (68\%) and SBF (64\%) (Fig. 1).

Cytological evaluation was more commonly used in cases of OE $(91 \%)$, followed by MD (88\%), SBF (83\%), and FD (72\%) (Fig. 2).

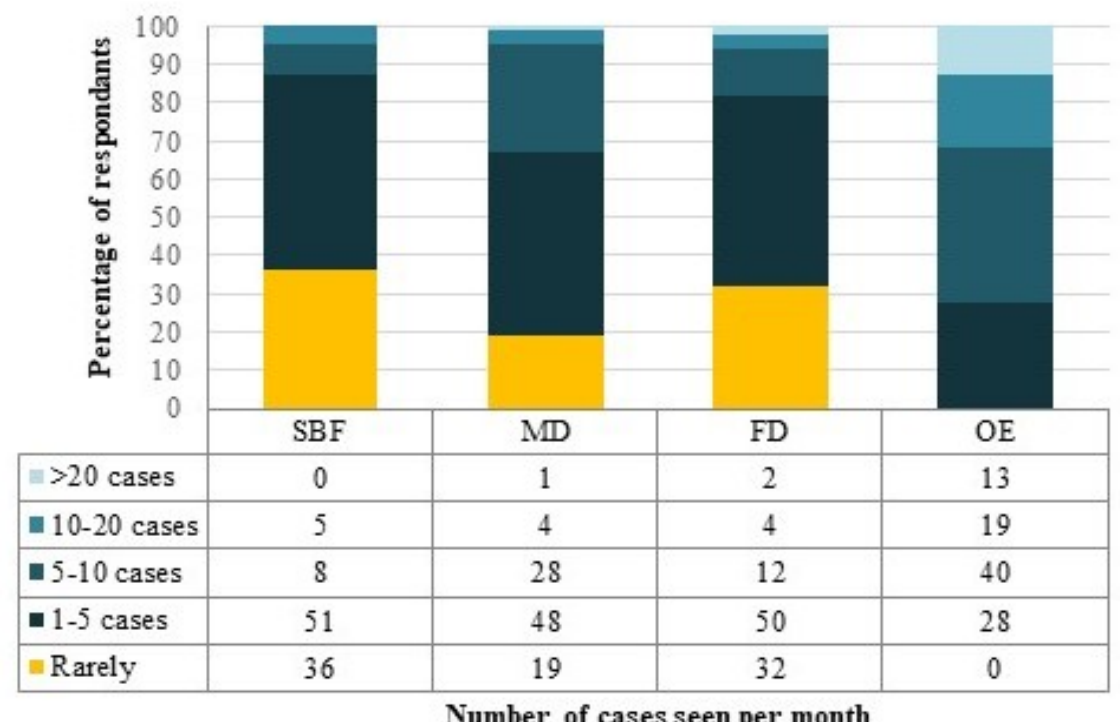

Fig. 1. Number of superficial bacterial folliculitis (SBF), Malassezia dermatitis (MD), fold dermatitis (FD), and otitis externa (OE) cases observed per month 


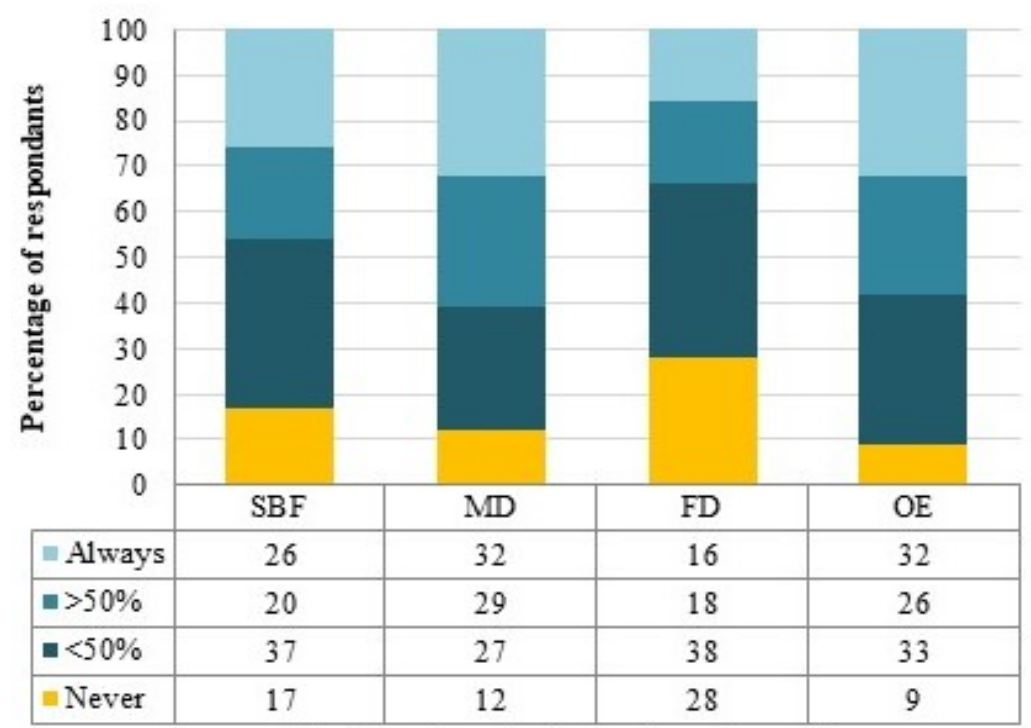

Number of cytologies performed per disease

Fig. 2. Cytological evaluation for diagnosis confirmation of superficial bacterial folliculitis (SBF), Malassezia dermatitis (MD), fold dermatitis (FD), and otitis externa (OE)



Fig. 3. Reasons for the use of bacterial culture and antibiotic susceptibility testing in cases of superficial bacterial folliculitis (SBF)

Presence of antibiotic resistance in SBF. All clinicians observed cases of SBF caused by antibioticresistant $S$. pseudintermedius. In fact, most clinicians (57\%) declared an increase in the number of antibioticresistant cases seen in the last five years, whereas 33\% did not think this was the case. Ten percent did not have an opinion on the prevalence of antibiotic resistance in $S$. pseudintermedius. Most clinicians treated SBF with empirical antibiotic therapy and only considered bacterial culture and antibiotic susceptibility testing after unsuccessful empirical treatment. Cases suspected at the initial stage of being aggravated by bacterial resistance were another reason for culture and antibiotic susceptibility, and in such cases this step was taken prior to treatment (Fig. 3).

\section{Treatment of bacterial infections}

Oral antibiotherapy. Oral antibiotics were frequently prescribed to manage infections due to S. pseudintermedius (Fig. 4). The results showed that SBF cases are very likely to be treated with oral antibiotics as $100 \%$ of the participants considered 
prescribing them in this circumstance. In FD and OE, the clinician still considered prescribing oral antibiotics in $88 \%$ and $82 \%$ of the cases, respectively.

For the treatment of SBF, amoxicillin with clavulanic acid was considered by all the clinicians. Cephalexin was also very commonly used (94\%), followed by enrofloxacin (67\%) and marbofloxacin $(60 \%)$. Antibiotics less commonly used were clindamycin (48\%), cefovecin (30\%), doxycycline (24\%), trimethoprim with sulfamethoxazole (22\%), and minocycline (10\%) (Fig. 5).

Topical therapy in bacterial infections. Participants prescribed therapeutic baths for SBF treatment followed by skin disinfection. The treatment was performed with topical antibiotics (either associated with antifungals or glucocorticoids). Fold dermatitis was managed by disinfection of the skin followed by topical antibiotics. Ear cleaning was frequently prescribed, and topical treatments containing a combination of antibiotic, antifungal, and glucocorticoids were the main choice. If honey-based products were considered, they would mainly be used for SBF and hardly used at all in the treatment of FD and OE. Other products (not specified) were also used by the participants (Table 1 ).



Fig. 4. Oral antibiotherapy use in superficial bacterial folliculitis (SBF), fold dermatitis (FD) and otitis externa (OE) due to S. pseudintermedius



Fig. 5. Use of oral antibiotherapy for superficial bacterial folliculitis: amoxicillin-clavulanic acid (AMC); cephalexin (CF); enrofloxacin (ENR); marbofloxacin (MAR); clindamycin (DA); cefovecin (CVN); doxycycline (DO); trimethoprimsulfamethoxazole (SXT) and minocycline (MH) 
Table 1. Topical therapy in superficial bacterial folliculitis (SBF), bacterial fold dermatitis (FD), and bacterial otitis externa (OE)

\begin{tabular}{|c|c|c|c|c|c|}
\hline Superficial bacterial folliculitis & Never & $<25 \%$ & $\begin{array}{l}25 \%- \\
50 \%\end{array}$ & $\begin{array}{l}50 \%- \\
75 \%\end{array}$ & $\begin{array}{l}75 \% \\
100 \%\end{array}$ \\
\hline Therapeutic baths & 0 & 4 & 18 & 14 & 64 \\
\hline Skin disinfection & 9 & 8 & 15 & 12 & 56 \\
\hline Product with antibiotic, antifungal and glucocorticoid & 43 & 31 & 15 & 8 & 3 \\
\hline Product with antibiotic only & 43 & 32 & 11 & 7 & 7 \\
\hline Honey-based products & 67 & 23 & 6 & 3 & 1 \\
\hline Other products & 69 & 17 & 6 & 4 & 4 \\
\hline \multicolumn{6}{|l|}{ Bacterial fold dermatitis } \\
\hline Skin disinfection & 0 & 3 & 2 & 15 & 80 \\
\hline Product with antibiotic, antifungal, and glucocorticoid & 29 & 26 & 8 & 21 & 16 \\
\hline Product with antibiotic only & 34 & 25 & 7 & 20 & 14 \\
\hline Honey-based product & 80 & 12 & 2 & 3 & 3 \\
\hline Other products & 72 & 12 & 1 & 8 & 7 \\
\hline \multicolumn{6}{|l|}{ Bacterial otitis externa } \\
\hline Ear cleaning & 3 & 0 & 6 & 4 & 87 \\
\hline Product with antibiotic, antifungal and glucocorticoid & 2 & 8 & 6 & 23 & 61 \\
\hline Product with antibiotic only & 54 & 24 & 10 & 9 & 3 \\
\hline Honey-based product & 92 & 6 & 2 & 0 & 0 \\
\hline Other products & 74 & 14 & 7 & 2 & 3 \\
\hline
\end{tabular}

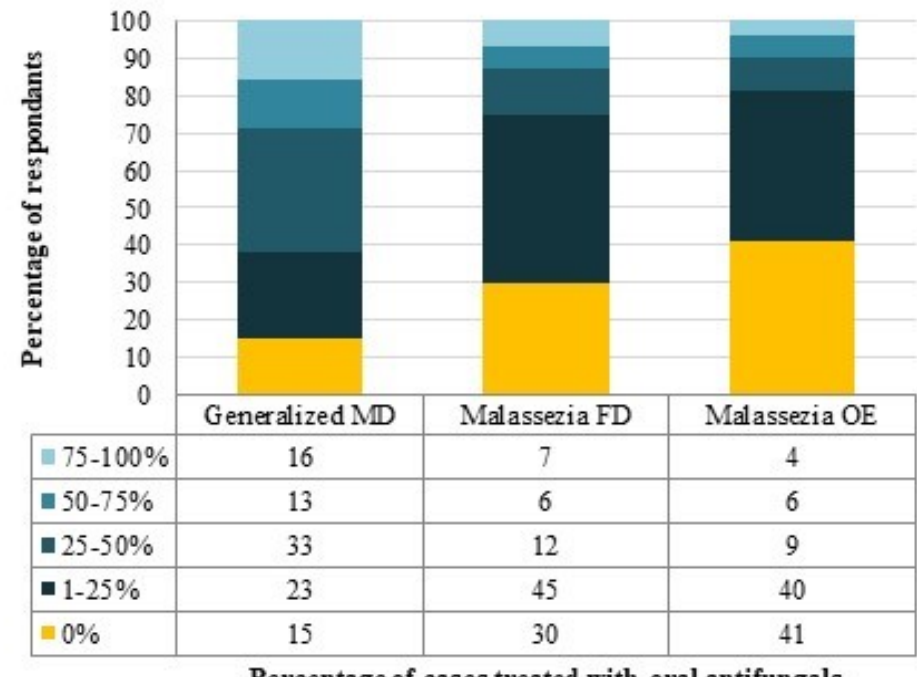

Percentage of cases treated with oral antifungals

Fig. 6. Oral antifungal use in generalised Malassezia dermatitis (MD), Malassezia fold dermatitis (FD), and Malassezia otitis externa (OE) cases

\section{Treatment of Malassezia infections}

Oral antifungals. For the treatment of generalised MD, oral antifungals were used by $85 \%$ of the clinicians. Concerning Malassezia FD and OE, oral antifungals were prescribed by $70 \%$ and $59 \%$ of the clinicians, respectively (Fig. 6).

Topical therapy in Malassezia infections. Topical treatment of generalised MD was performed with bathing and skin disinfection, and with products containing only antifungals. Malassezia FD was managed with skin disinfection followed by application of products containing antibiotic and antifungal agents and glucocorticoids. Ear cleaning followed by use of products with antibiotic and antifungal effect and glucocorticoid content was the treatment adopted for OE. Honey-based products are hardly used for any of the diseases caused by Malassezia. Other products that were not specified were also used by the clinicians (Table 2). 
Table 2. Use of topical therapy in Malassezia dermatitis (MD), fold dermatitis (FD), and otitis externa (OE)

\begin{tabular}{|c|c|c|c|c|c|}
\hline Generalised Malassezia dermatitis & Never & $<25 \%$ & $\begin{array}{l}25 \%- \\
50 \%\end{array}$ & $\begin{array}{l}50 \%- \\
75 \%\end{array}$ & $\begin{array}{l}75 \%- \\
100 \%\end{array}$ \\
\hline Therapeutic baths & 0 & 1 & 7 & 5 & 87 \\
\hline Skin disinfection & 20 & 12 & 7 & 12 & 49 \\
\hline Product with antibiotic, antifungal, and glucocorticoid & 53 & 21 & 13 & 10 & 3 \\
\hline Product with antifungal only & 36 & 17 & 19 & 12 & 16 \\
\hline Honey-based product & 96 & 3 & 0 & 1 & 0 \\
\hline Other products & 78 & 6 & 3 & 7 & 6 \\
\hline \multicolumn{6}{|l|}{ Malassezia fold dermatitis } \\
\hline Skin disinfection & 1 & 2 & 6 & 7 & 84 \\
\hline Product with antibiotic, antifungal, and glucocorticoid & 29 & 20 & 17 & 16 & 18 \\
\hline Product with antifungal only & 34 & 18 & 25 & 10 & 13 \\
\hline Honey-based product & 90 & 6 & 2 & 1 & 1 \\
\hline Other products & 74 & 11 & 5 & 5 & 5 \\
\hline \multicolumn{6}{|l|}{ Malassezia otitis externa } \\
\hline Ear cleaning & 4 & 1 & 3 & 8 & 84 \\
\hline Product with antibiotic, antifungal, and glucocorticoid & 7 & 6 & 12 & 15 & 59 \\
\hline Product with antifungal only & 61 & 12 & 9 & 10 & 8 \\
\hline Honey-based product & 95 & 2 & 2 & 0 & 1 \\
\hline Other products & 80 & 9 & 3 & 0 & 8 \\
\hline
\end{tabular}

\section{Discussion}

This study showed that largely oral antibiotics were used for the treatment of SBF, FD, and OE. Diagnostic approach is an issue, in veterinarians' failure to use of appropriate diagnostic tests for the conditions considered in the survey.

The majority of clinicians who collaborated in this survey were experienced in small animal practice and had been working for over 10 years. The conditions considered were observed routinely by the practitioners. This is in accordance with previous literature which states that $\mathrm{SBF}$ is a common disease and also one of the main reasons for antimicrobial prescription in small animal practice $(4,33)$. Otitis externa is also a common cause for consultation $(3,29$, 31). Malassezia dermatitis is another frequent disease and is normally associated with an underlying cause such as atopic dermatitis $(9,25)$. Skin fold dermatitis is also very common, particularly in brachycephalic dogs and breeds with excessive skin folds (5). Lately, brachycephalic breeds have become very common in Portugal, exemplified most clearly by the French Bulldog, and this might be the reason for the high prevalence of FD observed by the clinicians in this study.

In general, the survey demonstrated that cytology could be more thoroughly used by clinicians for diagnostic purposes. In fact, only approximately a quarter of the clinicians performed it in every case. Cytology is a simple, inexpensive, and reliable diagnostic test that can easily be performed in a consultation by the clinician (5). Unfortunately, 17\% of the clinicians never used this diagnostic tool for the diseases considered in this study, which is surprising, bearing in mind that adhesive cellophane testing is the most suitable alternative test for diagnosis of MD (7). Otic cytology allows discrimination between bacteria and Malassezia yeasts, and therefore is superior to the adhesive cellophane tape test as the appearance and odour of ear exudate adhering to tape cannot be used to reach a reliable diagnosis $(2,21)$.

Clinicians always considered the use of oral antibiotherapy in SBF cases. Overall, clinicians preferred to begin therapy with oral antibiotics, empirically, and if clinical improvement was not observed, they resorted to bacterial culture and susceptibility testing. When bacterial resistance is suspected, the clinicians will also perform culture and susceptibility testing. There are situations when bacterial culture is particularly important, mainly in cases of apparent antimicrobial resistance (18).

However, the number of clinicians who never used bacterial culture, never tested for antibiotic susceptibility, or only used culture for diagnostic purposes is surprising. In fact, most of the participants had diagnosed cases of SBF with antibiotic resistance to $S$. pseudintermedius and had recognised an increase in antibiotic resistance in the last five years. The problem of their diminishing effectiveness is therefore escalating. In Portugal, methicillin and multidrug resistant $S$. pseudintermedius were reported for the first time in 2010 and in other papers thereafter $(6,11$, 12, 32).

Bacterial FD and $\mathrm{OE}$ were also largely treated with oral antibiotics, which adds further concern. 
Exposure to antibiotics has been associated with the development of resistance by $S$. pseudintermedius isolates, either from skin lesions or from $\operatorname{OE}(18,23$, $35,36)$.

This survey showed that amoxicillin-clavulanic acid and cephalexin were the most frequently prescribed antibiotics for SBF treatment, followed by enrofloxacin and marbofloxacin. Other antibiotics such as clindamycin, trimethoprim-sulfamethoxazole, cefovecin, doxycycline, and minocycline were less frequently used. This is in accordance with official data: penicillins, first and second generation cephalosporins, and fluoroquinolones are the most prescribed antibiotics in small animal practice (15). Macrolides and tetracyclines are less often used, as well as sulphonamides and lincosamides (15). A 16year study in Portugal documented an increase in $S$. pseudintermedius resistance against oxacillin, ampicillin, amoxicillin, penicillin, cefovecin, cefalexin, enrofloxacin, clindamycin, and trimethoprimsulfamethoxazole. Cephalosporins have been putatively implicated in the development of MRSP (13). Another study reports misuse of antimicrobials such as fluoroquinolones, macrolides, and third generation cephalosporins, demonstrating correlation with MRSP colonisation (34). Based on the results of this survey, we recommend that fluoroquinolones should be used with more caution. Clinicians principally use antibiotics in the treatment of SBF, FD, and $\mathrm{OE}$, in spite of the conservative recommendations of the ISCAID guidelines. The guidelines developed by ISCAID are a great asset to help the clinician recognise the signs of canine SBF, choose the correct diagnostic tools, and determine the most appropriate topical or systemic antimicrobial therapy (18). In reality, most of the practitioners do not follow or are not aware of the ISCAID guidelines, although the reasons are not explained in this survey.

In general, all clinicians recommended the application of therapeutic baths and skin disinfection in cases of bacterial or Malassezia infections. The use of antibiotic and antimycotic based-products was also frequent, in contrast to honey-based products which were rarely applied.

According to our findings, topical treatment with honey-based products is seldom prescribed. The efficacy of a honey-based gel was also confirmed for the treatment of bacterial and/or Malassezia otitis externa and canine intertrigo $(19,26)$. The same product has been proven to be effective against MSSP and MRSP originating from SBF cases. The product also eradicated $M$. pachydermatis originating from $\mathrm{OE}$ (30). Medical honey or honey-based products are potential treatments for the diseases considered in this study and could be used more often in Portugal.

This survey contributed to understanding how Portuguese veterinarians are diagnosing and treating superficial bacterial folliculitis, Malassezia dermatitis, fold dermatitis, and otitis. It uncovered a lack of awareness of the ISCAID guidelines, an increasing perception of antibiotic-resistant $S$. pseudintermedius, potential overuse of antibiotics, and lack of antibioticfree products. Educational actions should be undertaken to increase awareness about the correct use of antibiotics to avoid promulgating bacterial resistance in our country.

Conflict of Interests Statement: The authors declare that there is no conflict of interests regarding the publication of this article.

Financial Disclosure Statement: This study was selffunded.

Animal Rights Statement: None required.

\section{References}

1. Allaker R.P., Lloyd D.H., Simpson A.I.: Occurrence of Staphylococcus intermedius on the hair and skin of normal dogs. Res Vet Sci 1992, 52, 174-176.

2. Angus J.C.: Otic cytology in health and disease. Vet Clin North Am Small Anim Pract 2004, 34, 411-424.

3. August J.R.: Otitis externa. A disease of multifactorial etiology. Vet Clin North Am Small Anim Pract 1998, 18, 731-742.

4. Baker S.A., Van-Balen J., Lu B., Hillier A., Hoet A.E.: Antimicrobial drug use in dogs prior to admission to a veterinary teaching hospital. J Am Vet Med Assoc 2012, 241, 210-217.

5. Beco L., Guaguère E., Méndez C.L., Noli C., Nuttall T., Vroom M.: Suggested guidelines for using systemic antimicrobials in bacterial skin infections: part 1 - diagnosis based on clinical presentation, cytology, and culture. Vet Rec 2013, 172, 72-78.

6. Beça N., Bessa L.J., Mendes A., Santos J., Leite-Martins L., Matos A.J., da Costa P.M.: Coagulase-positive Staphylococcus: prevalence and antimicrobial resistance. J Am Anim Hosp Assoc 2015, 51, 365-371.

7. Bensignor E., Jankowski F., Seewald W., Touati F., Deville M., Guillot J.: Comparison of two sampling techniques to assess quantity and distribution of Malassezia yeasts on the skin of Basset hounds. Vet Dermatol 2000, 213, 237-241.

8. Bryan J., Frank L.A., Rohrbach B.W., Burgette L.J., Cain C.L., Bemis D.A.: Treatment outcome of dogs with meticillin-resistant and meticillin-susceptible Staphylococcus pseudintermedius pyoderma. Vet Dermatol 2012, 23, 361-368.

9. Bond R., Ferguson E.A., Curtis C.F., Craig J.M., Lloyd D.G.: Factors associated with elevated cutaneous Malassezia pachydermatis populations in dogs with pruritic skin disease. J Small Anim Pract 1996, 37, 103-107.

10. Cafarchia C., Iatta R., Immediato D., Puttilli M.R., Otranto D.: Azole susceptibility of Malassezia pachydermatis and Malassezia furfur and tentative epidemiological cut-off values. Med Mycol 2015, 53, 743-748.

11. Couto N., Pomba C., Moodley A., Guardabassi L.: Prevalence of meticillin-resistant staphylococci among dogs and cats at a veterinary teaching hospital in Portugal. Vet Rec 2011, 169,72 .

12. Couto N., Belas A., Couto I., Perreten V., Pomba C.: Genetic relatedness, antimicrobial and biocide susceptibility comparative analysis of meticillin-resistant and -susceptible Staphylococcus pseudintermedius from Portugal. Microb Drug Resist 2014, 20, 364-371.

13. Couto N., Monchique C., Belas A., Marques C., Gama L.T., Pomba C.: Trends and molecular mechanisms of antimicrobial resistance in clinical staphylococci isolated from companion 
animals over a 16 year period. J Antimicrob Chemother 2016, 71, 1479-1487.

14. Detwiler A., Bloom P., Petersen A., Rosser E.J.: Multi-drug and methicillin resistance of staphylococci from canine patients at a veterinary teaching hospital (2006-2011). Vet Q 2013, 33, 60-67.

15. DGAV (2015) Sales of veterinary antimicrobial agents in 30 European countries in 2015. Seventh ESVAC Report https://www.dgv.min-agricultura.pt/.

16. Fazakerley J., Nuttall T., Sales D., Schmidt V., Carter S.D., Hart C.A., McEwan N.A.: Staphylococcal colonization of mucosal and lesional skin sites in atopic and healthy dogs. Vet Dermatol 2009, 20, 179-184.

17. Ganiere J.P., Medaille C., Mangion C.: Antimicrobial drug susceptibility of Staphylococcus intermedius clinical isolates from canine pyoderma. J Vet Med B Infect Dis Vet Public Health 2005, 52, 25-31.

18. Hillier A., Lloyd D.H., Weese J.S., Blondeau J.M., Boothe D., Breitschwerdt E., Guardabassi L., Papich M.G., Rankin S., Turnidge J.D., Sykes J.E.: Guidelines for the diagnosis and antimicrobial therapy of canine superficial bacterial folliculitis (Antimicrobial Guidelines Working Group of the International Society for Companion Animal Infectious Diseases). Vet Dermatol 2014, 25, 163-175.

19. Jakobsson Z.: Pilotstudie för att utvärdera effekten av L-Mesitran Honungsbaserad sårsalva - vid behandling av yt-pyodermi hos hund (Pilot study to evaluate the effect of L-Mesitran Honeybased wound ointment - in the treatment of surface pyoderma in dogs). Fakulteten för Veterinärmedicin och husdjursvetenskap, Uppsala, Sweden, 2011. https://stud.epsilon. slu.se/2224/1/jakobsson_z_110131.pdf.

20. Jesus F.P., Lautert C., Zanette R.A., Mahl D.L., Azevedo M.I., Machado M.L., Dutra V., Button S.A., Alves S.H., Santurio J.M.: In vitro susceptibility of fluconazole-susceptible and resistant isolates of Malassezia pachydermatis against azoles. Vet Microbiol 2011, 152, 161-164.

21. Kiss G., Radványi S., Szigeti G., Lukáts B., Nagy G.: New combination for the therapy of canine otitis externa. I. Microbiology of otitis externa. J Small Anim Pract 1997, 38 , 51-56.

22. Loeffler A., Linek M., Moodley A., Guardabassi L., Sung J.M., Winkler M., Weiss R., Lloyd D.H.: First report of multiresistant, mecA-positive Staphylococcus intermedius in Europe: 12 cases from a veterinary dermatology referral clinic in Germany. Vet Dermatol 2007, 18, 412-421.

23. Ludwig C., de Jong A., Moyaert H., El Garch J., Janes R., Klein U., Morrissey I., Thiry J., Youala M.: Antimicrobial susceptibility monitoring of dermatological bacterial pathogens isolated from diseased dogs and cats across Europe (ComPath results). J Appl Microbiol 2016, 121, 1254-1267.

24. Lyskova P., Vydrzalova M., Mazurova J.: Identification and antimicrobial susceptibility of bacteria and yeasts isolated from healthy dogs and dogs with otitis externa. J Vet Med A Physiol Pathol Clin Med 2007, 54, 559-563.

25. Machado M.L., Ferreiro L., Ferreira R.R., Corbellini L.G., Deville M., Berthelemy M., Guillot J.: Malassezia dermatitis in dogs in Brazil: diagnosis, evaluation of clinical signs, and molecular identification. Vet Dermatol 2011, 22, 46-52.

26. Maruhashi E., Braz B.S., Nunes T., Pomba C., Belas A., DuarteCorreia J.G., Lourenço A.M.: A pilot study on the use of medical honey in the management of canine otitis externa. $28^{\text {th }}$ Annual Congress of the European Society and College of Veterinary Dermatology, Krakow, Poland, 2015, p. 118.

27. Morris D.O.: Malassezia dermatitis and otitis. Vet Clin North Am Small Anim Pract 1999, 29, 1303-1310.

28. Nijima M., Kano R., Nagata M., Hasegawa A., Kamata H.: An azole-resistant isolate of Malassezia pachydermatis. Vet Microbiol 2011, 49, 288-290.

29. O’Neill D.G., Church D.B., McGreevy P.D., Thomson P.C., Brodbelt D.C.: Prevalence of disorders recorded in dogs attending primary-care veterinary practices in England. PloS One 2014, 9, e90501.

30. Oliveira A.M., Devesa J.S., Hill P.B.: In vitro efficacy of a honey based gel against canine clinical isolates of Staphylococcus pseudintermedius and Malassezia pachydermatis. Vet Dermatol 2018, 29, 180-e65.

31. Perry L.R., MacLennan B., Korven R., Rawlings T.A.: Epidemiological study of dogs with otitis externa in Cape Breton, Nova Scotia. Can Vet J 2017, 58, 168-174.

32. Pomba C., Couto N., Moodley A.: Treatment of a lower urinary tract infection in a cat caused by a multi-drug methicillinresistant Staphylococcus pseudintermedius and Enterococcus faecalis. J Feline Med Surg 2010, 12, 802-806.

33. Rantala M., Lahti E., Kuhalampi J., Pesonen S., Jarvinen A.K., Saijonmaa-Koulumies L., Honkanen-Buzalski T.: Antimicrobial resistance in Staphylococcus spp., Escherichia coli, and Enterococcus spp. in dogs given antibiotics for chronic dermatological disorders, compared with non-treated control dogs. Acta Vet Scand 2004, 45, 37-45.

34. Rota A., Milani C., Corrò M., Drigo I., Börjesson S.: Misuse of antimicrobials and selection of methicillin-resistant Staphylococcus pseudintermedius strains in breeding kennels: genetic characterization of bacteria after a two-year interval. Reprod Domest Anim 2013, 48, 1-6.

35. Weese J.S., Faires M.C., Frank L.A., Reynolds L.M., Battisti A.: Factors associated with methicillin-resistant versus methicillinsusceptible Staphylococcus pseudintermedius infection in dogs. J Am Vet Med Assoc 2012, 240, 1450-1455.

36. Zur G., Gurevich B., Elad D.: Prior antimicrobial use as a risk factor for resistance in selected Staphylococcus pseudintermedius isolates from the skin and ears of dogs. Vet Dermatol 2016, 27, 468-e125. 